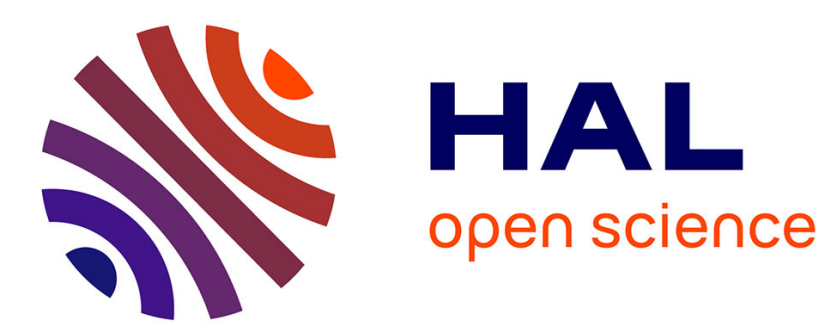

\title{
Dynamic reorganization of functional brain networks during picture naming.
}

Mahmoud Hassan, Pascal Benquet, Arnaud Biraben, Claude Berrou, Olivier Dufor, Fabrice Wendling

\section{- To cite this version:}

Mahmoud Hassan, Pascal Benquet, Arnaud Biraben, Claude Berrou, Olivier Dufor, et al.. Dynamic reorganization of functional brain networks during picture naming.. Cortex, 2015, 73, pp.276-288. 10.1016/j.cortex.2015.08.019 . hal-01222833

\section{HAL Id: hal-01222833 \\ https://hal.science/hal-01222833}

Submitted on 30 Oct 2015

HAL is a multi-disciplinary open access archive for the deposit and dissemination of scientific research documents, whether they are published or not. The documents may come from teaching and research institutions in France or abroad, or from public or private research centers.
L'archive ouverte pluridisciplinaire HAL, est destinée au dépôt et à la diffusion de documents scientifiques de niveau recherche, publiés ou non, émanant des établissements d'enseignement et de recherche français ou étrangers, des laboratoires publics ou privés. 
Dynamic reorganization of functional brain networks during picture naming

Mahmoud Hassan ${ }^{1,2}$, Pascal Benquet ${ }^{1,2}$, Arnaud Biraben ${ }^{1,2,3}$, Claude Berrou ${ }^{4,5}$, Olivier Dufor ${ }^{4,5}$ and Fabrice Wendling ${ }^{1,2}$

${ }^{1}$ INSERM, U1099, Rennes, F-35000, France

${ }^{2}$ Université de Rennes 1, LTSI, F-35000, France

${ }^{3}$ Neurology dpt, CHU, Rennes, France

${ }^{4}$ Télécom Bretagne (Institut Mines-Télécom), UMR CNRS Lab-STICC, Brest, France

${ }^{5}$ UMR CNRS Lab-STICC, Brest, France

Corresponding author:

Mahmoud Hassan

mahmoud.hassan@univ-rennes1.fr

Tel: +33223235605 / 6220

Fax: +33223236917

Hassan, M., Benquet, P., Biraben, A., Berrou, C., Dufor, O., Wendling, F., 2015a. Dynamic reorganization of functional brain networks during picture naming. Cortex 73, 276-288. 


\section{Abstract}

For efficient information processing during cognitive activity, functional brain networks have to rapidly and dynamically reorganize on a sub-second time scale. Tracking the spatiotemporal dynamics of large scale networks over this short time duration is a very challenging issue. Here, we tackle this problem by using dense electroencephalography (EEG) recorded during a picture naming task. We found that (i) the picture naming task can be divided into six brain network states (BNSs) characterized by significantly high synchronization of gamma $(30-45 \mathrm{~Hz})$ oscillations, (ii) fast transitions occur between these BNSs that last from $30 \mathrm{~ms}$ to $160 \mathrm{~ms}$, (iii) based on the state of the art of the picture naming task, we consider that the spatial location of their nodes and edges, as well as the timing of transitions, indicate that each network can be associated with one or several specific function (from visual processing to articulation) and (iv) the comparison with previously-used approach aimed at localizing the sources showed that the network-based approach reveals networks that are more specific to the performed task. We speculate that the persistence of several brain regions in successive BNSs participates to fast and efficient information processing in the brain. 


\section{Introduction}

Any cognitive process involves the activation of a large-scale functional brain network (Bressler \& Menon, 2010). In visual, attentional and memory processes, this network is characterized by increased synchronization of cortical oscillations (in the gamma frequency range (Doesburg, Roggeveen, Kitajo, \& Ward, 2008), in particular but not only) across distant neuronal assemblies distributed over distinct brain areas.

The accurate tracking of the spatiotemporal dynamics of large-scale networks over the duration (often as short as a few hundreds of ms) of cognitive processes is still a challenging issue (Allen et al., 2012; Hutchison et al., 2013). A number of theories have been elaborated to explain these spatiotemporal dynamics. It has been hypothesized that functional brain networks engage in fast transitions between transiently stable states, each characterized by a network with intrinsic dynamics and with specific functional relationships between neuronal assemblies (Hansen, Battaglia, Spiegler, Deco, \& Jirsa, 2014; Sporns, 2010). According to this theory, the substrate of cognitive processes would correspond to a sequence of switches between networks and, thus, to time- and space-dependent fluctuations in the node and edge properties of the global network.

The validation of such hypotheses for task-related data requires the following of brain processes at the millisecond time-scale. This can barely be achieved using fMRI data for a simple and well-known reason: although they are characterized by an excellent spatial resolution, BOLD signals reflect the metabolic and hemodynamic response of neuronal assemblies (at voxel level). This slow response (seconds) is obviously related to the fast dynamics of cortical oscillations taking place over interconnected neuronal assemblies and 
defining functional networks, but indirectly (i.e. through the neuro-glial-vascular coupling (Logothetis, Pauls, Augath, Trinath, \& Oeltermann, 2001)).

In this study, we address this issue using EEG source connectivity analysis to track the spatiotemporal dynamics of large-scale networks associated with cognitive activity. We collected dense-EEG data from 21 subjects performing a picture naming task. We then reconstructed the functional networks in both their spatial and temporal dimension, over the entire duration of the cognitive process (from image perception to motor response) using a recently reported method (Hassan, Dufor, Merlet, Berrou, \& Wendling, 2014) that combines i) the solution to the inverse EEG problem, ii) the estimation of brain connectivity from phase locking values and iii) the segmentation of functional networks using a clustering method (Mheich, Hassan, Khalil, Berrou, \& Wendling, 2015) (see figure 1A). Our results reveal that the cognitive process can be decomposed into a sequence of transiently-stable and partiallyoverlapping networks. We assume, based on the state of the art of the picture naming task, that each network might be associated with a specific function (Levelt, Praamstra, Meyer, Helenius, \& Salmelin, 1998) (visual percept computing, lexical concept activation, selecting the target word from the mental lexicon, phonological encoding, phonetic encoding, and initiation of articulation) of the whole cognitive process. The results show that dense-EEG can bring highly valuable information about cortical networks, with both high spatial (1000 cortical regions) and temporal (ms time-scale) resolution. We speculate that the identified brain network states (BNSs) contribute to fast and efficient information processing in the brain. 


\section{Materials and methods}

\section{Picture naming task}

Twenty one right-handed healthy volunteers (11 women: mean age 28 year; min: 19, max: 40 and 10 men: mean age 23 years; min: 19, max: 33), with no neurological disease, were involved in this study. Participants were asked to name at a normal speed 148 displayed pictures on a screen using E-Prime 2.0 software (Psychology Software Tools, Pittsburgh, PA) (Schneider, Eschman, \& Zuccolotto, 2002). The images were selected from a database of 400 pictures standardized for French (Alario \& Ferrand, 1999) and were used during session about eight minute. They were controlled according to several parameters (see table S1). All pictures were shown as black drawings on a white background. Order of presentation was randomized across participants. Naming latencies were determined as the time between picture onset and the beginning of vocalization recorded by the system. Oral responses were recorded and then analyzed with Praat software to set the voice onset time (Boersma, 2002). This study was approved by the National Ethics Committee for the Protection of Persons (CPP), conneXion study, agreement number (2012-A01227-36), and promoter: Rennes University Hospital. All participants provide their written informed consent to participate in this study. The ethics committee has approved the consent procedure. A typical trial started with the appearance of an image during 3 seconds followed by a jittered inter-stimulus interval of 2 or 3 seconds randomly. Most responses were given while the image was still present on the screen. Errors in naming were discarded for the subsequent analysis. A total of 
2926 on 3108 events were considered. The fastest response time delay for an event was $<600$ ms (see figure S1 for the responses delays of all subjects).

\section{Data}

The brain activity was recorded using dense-EEG, 256 electrodes, system (EGI, Electrical Geodesic Inc.). The main feature of this system is the large coverage of the subject's head by surface electrodes allowing for the improved analysis of the intracerebral activity from noninvasive scalp measurements, as compared with 32 -to 128- electrodes standard systems. EEG signals were collected with a $1 \mathrm{kHz}$ sampling frequency and band-pass filtered between 3 and 45Hz. Each trial was visually inspected, and epochs contaminated by eye blinking, movements or any other noise source were rejected and excluded from the analysis performed using the EEGLAB open source toolbox (Delorme \& Makeig, 2004).

\section{EEG source connectivity}

A crucial step when realizing EEG source connectivity analysis is the choice of three factors: the method used to solve the inverse problem, the method used to compute the functional connectivity among the time series of the reconstructed sources and the number of electrodes used on the scalp. Very recently, we have described a comparative study of these factors and we showed that a combination of the weighted Minimum Norm Estimate (wMNE) with the Phase Locking Value (PLV) using high resolution EEG is the best combination among the tested combination (Hassan et al., 2014). This combination was used in the presented work.

According to the linear discrete equivalent current dipole model, EEG signals $\mathbf{S}(\mathrm{t})$ measured from Q channels can be expressed as linear combinations of P time-varying current dipole sources $\mathbf{D}(\mathrm{t})$ :

$$
\mathbf{S}=\mathrm{G} . \mathbf{D}+\mathbf{B}
$$


where $\mathbf{G}$ and $\mathbf{B}(\mathrm{t})$ are respectively the matrix containing the lead fields of the dipolar sources and the additive noise. In the general case, the inverse problem consists in finding an estimate $\hat{\mathbf{D}}(\mathrm{t})$ of the dipolar source parameters (typically, the position, orientation and magnitude), given the EEG signals $\mathbf{S}(\mathrm{t})$ and given the gain matrix $\mathbf{G}$. This matrix can be computed from a multiple layer head model (volume conductor) and from the position of electrodes. For instance, the Boundary Element Method is a numerical method classically used in the case of realistic head models.

As this problem is ill-posed $(\mathrm{P}>>\mathrm{Q})$, physical and mathematical constraints have to be added to obtain a unique solution among the many solutions that minimize the residual term in the fitting of measured EEG signals. Using segmented MRI data, the source distribution can be constrained to a field of current dipoles homogeneously distributed over the cortex (Dale \& Sereno, 1993), and normal to the cortical surface.

Technically, in the source model, we assumed that EEG signals are generated by macrocolumns of pyramidal cells lying in the cortical mantle and aligned orthogonally with respect to its surface (Nunez, 2006). Thus, the electrical contribution of each macro-column to scalp electrodes can be represented by a current dipole located at the center of gravity of each triangle of the 3D mesh and oriented normally to the triangle surface. Using this source space, the weighted Minimum Norm Estimate (wMNE) method only estimates the moment of dipole sources. The wMNE compensates for the tendency of classical MNE to favor weak and surface sources. This is done by introducing a weighting matrix $\mathbf{W}_{\mathbf{s}}$ :

$$
\hat{D}_{\text {wMNE }}=\left(G^{T} W_{S} G+\lambda I\right)^{-1} G^{T} W_{S} S
$$

where matrix $\mathbf{W}_{\mathbf{S}}$ adjusts the properties of the solution by reducing the bias inherent to MNE solutions. Classically, $\mathbf{W}_{\mathbf{S}}$ is a diagonal matrix built from matrix $\mathbf{G}$ with non-zero terms inversely proportional to the norm of the lead field vectors. The value of $\lambda$ is computed 
relatively to the signal to noise ratio for each signal computed as the ration between the poststimuli period to the pre-stimulus $(200 \mathrm{~ms})$. The $\lambda$ value was between 0.1 and 0.3 .

The sources were reconstructed for each trial (same number of sources for each trial) and the functional connectivity is then computed between the reconstructed sources using the phase synchronization (PS) method. The first step for estimating the PS is to extract the instantaneous phase of each signal. We used the method based on Hilbert transform in our study. The second step is the definition of an appropriate index to measure the degree of synchronization between estimated instantaneous phases. To measure PS, the phase locking value (PLV) method is used as described in (Lachaux, Rodriguez, Martinerie, \& Varela, 1999). For each source pair, $x$ and $y$, at time $t\left(t=t_{1}, \ldots, t_{T}\right.$ where $T=D * f_{\mathrm{s}} ; D$ and $f_{\mathrm{s}}$ denote the signal length relative to the onset and the sampling frequency, respectively) for the $\operatorname{Tr}$ trials and for subject $j(j=1 \ldots M$, where $M$ denotes the number of subjects), PLV is defined as:

$$
\operatorname{PLV}_{x y}^{j}(t)=\frac{1}{T r}\left|\sum_{n=1}^{N} \varphi_{x}(t)-\varphi_{y}(t)\right|
$$

To reduce the effect of correlations between near electrodes we apply a normalization procedure (z-score) so that the $\mathrm{PLV}_{x y}$ values were compared with the $200 \mathrm{~ms}$ baseline preceding the presentation of the image. Let $\mu_{x y}$ and $\sigma_{x y}$ are the mean and standard deviation computed from a $200 \mathrm{~ms}$ pre-stimulus baseline. The normalized PLVs are then defined as $\overline{\operatorname{PLV}}_{x y}^{j}(t)=\left(\operatorname{PLV}_{x y}^{j}(t)-\mu_{x y}^{j}\right) / \sigma_{x y}^{j}$. The functional connectivity was computed at the low gamma band $(30-45 \mathrm{~Hz})$. This frequency band is the most relevant one in the context of the similar cognitive task performed by the subjects, as reported in (Fell \& Axmacher, 2011; Rodriguez et al., 1999; Supp, Schlögl, Trujillo-Barreto, Müller, \& Gruber, 2007). The PLVs were then averaged over subjects: 


$$
\overline{\operatorname{PLV}}_{x y}(t)=\frac{1}{M} \sum_{j=1}^{M} \operatorname{PLV}_{x y}^{\mathrm{j}}(t)
$$

where $\overline{\operatorname{PLV}}_{x y}(t)$ represents the general term of the average adjacency matrix $\overline{\operatorname{PLV}}(t)$ which defines a functional connectivity network $\mathrm{N}$ at each time $t, N=\{N(t), t=1, . ., T\}$, computed for the $V$ pairs of $x$ and $y$ sources, where $V$ is equal to $(N c .(N c-1) / 2))$ and $N c$ is the number of ROIs.

\section{Functional connectivity states}

We recently developed an algorithm to decompose cognitive task into functional connectivity states (Mheich et al., 2015). The objective of this algorithm is to identify clusters among the $T$ networks $N(t)$. The proposed algorithm is based on the $K$-means clustering of the connectivity networks obtained by the PLV method. This approach allowed us to summarize brain networks into a limited number of dominant networks over given time period.

Briefly, the averaged connectivity matrices over all subjects were first obtained. $K$ networks (varies from 3 to 12 ) were then randomly selected and the spatial correlations between the $K$ networks and all the $T$ networks were computed. This gives a spatial correlation value for each $K$ at each time instant and for any of the $T$ networks only one of the $K$ networks produces the highest spatial correlation. Finally, the cross-validation criterion, first introduced by (Pascual-Marqui, Michel, \& Lehmann, 1995), was used to determine the optimal number of networks that explained the best the ongoing cognitive task. To investigate the inter-subject variability, we added an index called 'network presence' which calculate the ratio (in \%) of the identified networks/clusters among all the subjects (see Supplementary document for detailed description of the algorithm). 


\section{Statistical tests}

The segmentation algorithm based on $k$-means, produces a number of functional connectivity states (clusters). We assume that these clusters reflect changes in the cognitive state. To verify this assumption, we compare our results with an appropriate null model. The identified clusters are compared with those obtained by shuffling the original data using surrogates (procedure detailed in (Hlinka \& Hadrava, 2015)). Briefly, we use multivariate Fourier transform surrogates $\left(\mathrm{n}_{\text {sur }}=100\right)$ generated from the original EEG data for all trials. Such surrogates correspond to realizations of linear stationary process with conserved auto-and cross-correlation characteristics. The same processing steps were performed on these surrogates as on the original data: filtering in the gamma band: $30-45 \mathrm{~Hz}$, computing the functional connectivity using phase locking values at this frequency band, thresholding the connectivity matrices and segmenting into clusters using $k$-means algorithm. We then compared the spatial distributions ( $\mathrm{Sd})$ and the temporal profiles $(\mathrm{Tp})$ of the original clusters with those obtained using surrogates.

Concerning the $\mathrm{Tp}$, when a cluster is significant (i.e. related to the cognitive process and not to chance), the value of $\mathrm{Tp}$ for the surrogate data $\left(\mathrm{Tp}_{\mathrm{surr}}\right)$ will be different than the one of the original cluster $\left(\mathrm{T} \mathrm{p}_{\text {org }}\right)$. The underlying null hypothesis is that the obtained clusters are significantly different from the spurious networks states that can be always observed in random data or white noise (Hlinka \& Hadrava, 2015). The null hypothesis is tested by comparing the $T \mathrm{p}_{\text {surr }}$ and $\mathrm{T} \mathrm{p}_{\text {org }}$ using a statistical test. The "Rank test" is used to reject or accept the null hypothesis. Basically, $\left[\mathrm{Tp}_{\mathrm{org}} ; \mathrm{T} \mathrm{p}_{\text {surr }}\right]$ is sorted in increasing order and the rank index for $T p_{\text {org }}$ is returned. With a number of surrogates ( $\mathrm{n} \_$surr $=100$ for example), if this rank is $>95$ and $<5$ (significance level at 95\%), this means that it lies in the tail of the distribution, and that the null hypothesis can be rejected (two-tailed test) with a significance 
of $p=2 *(1 /($ n_surr +1$))=0.019$. A similar test is used for Sd. For multiple tests and to deal with the family wise error rate (FWER), we used the well-known Bonferroni correction test, which is considered as the simplest and most conservative method to control the FWER problem

\section{Regions of interest and network measures}

We used Freesurfer (Fischl, 2012) to register a labeled mesh from an average brain, where each label corresponded to one of 148 anatomical cortical regions (Destrieux, Fischl, Dale, \& Halgren, 2010). This output provided a standardized partition of the cortex into 148 regional areas. Each of these areas was then subdivided into a set of small sub-regions using Brainstorm (Tadel, Baillet, Mosher, Pantazis, \& Leahy, 2011), resulting in 1000 ROIs covering the whole cortex. This segmentation provided us with high resolution connection matrices (see figure 1A). These ROIs were then considered as In order to obtain a sparse, weighted, undirected graph, the adjacency matrices were thresholded. For each matrix, 10000 edges were retained. All corresponding weight values were positive. Then, the strength measure was used to characterize the nodes in the obtained weighted networks. This measure is defined as the sum of all edge weights for each node. For any node $i$, the strength $k_{i}^{w}$ is defined as:

$$
k_{i}^{w}=\sum_{j \in N} W_{i j}
$$

where $N$ is the set of all nodes in the graph and $W_{i j}$ is the connection weight between two nodes $i$ and $j$. This measure was computed using the Brain Connectivity Toolbox (BCT) (Rubinov \& Sporns, 2010). 


\section{Software}

We used MATLAB ${ }^{\circledR}(2007 \mathrm{a}$, MathWorks Inc.) in the entire process: from EEG preprocessing, source reconstruction, functional connectivity analysis, and computation of the graph parameters and visualization of the brain networks. These processing steps were carried out by using several tools mainly Freesurfer http://surfer.nmr.mgh.harvard.edu/ for surface parcellation, Brainstorm http://neuroimage.usc.edu/brainstorm/ for source reconstruction, EEGLAB http://sccn.ucsd.edu/eeglab/ for EEG preprocessing, and other homemade codes. 3D brain networks were visualized using a recently developed tool in our team called EEGNET. The network measures were computed from the brain connectivity toolbox (BCT) https://sites.google.com/site/bctnet/ that was integrated in EEGNET.

\section{Results}

Functional connectivity states identified from dense-EEG recordings. We have recently developed a new clustering algorithm to detect the brain network states (BNSs) at millisecond scale from dense EEG recordings (Mheich et al., 2015) (figure 1B). A BNS is defined by a transiently-stable brain network in which nodes are associated with distributed neuronal assemblies and in which edges denote significantly high phase synchronization of their oscillatory activity in the gamma band (30-45 Hz) (see Materials and Methods for details about the algorithm). In order to assess brain dynamics during information processing (from perception to behavioral response), this algorithm was applied to high-resolution group averaged connectivity matrices obtained from dense EEG signals recorded in 21 subjects performing a picture naming task.

Results are shown in figure 2 and they revealed that the cognitive process could be divided into 6 BNSs. To globally characterize the networks for each of these 6 BNSs, we show the 
number of nodes for five macroscopic regions (occipital $(\mathrm{O})$, parietal $(\mathrm{P})$, temporal $(\mathrm{T})$, frontal (F) and central (C)) expressed in percent (with respect to the total number of nodes).

The first BNS corresponds to the time period ranging from 0 (stimulus onset) to $119 \mathrm{~ms}$. This network mainly involves the occipital pole (L: 62\%, R: 64\%), the temporal lobe (L: $30 \%$ and R: $14 \%$ ) and the parietal lobe (L: 1\% and R: 22\%). A second stable state (BNS2) was then observed between 120 and $150 \mathrm{~ms}$ where the network is mainly located in the occipital (L: 88\%, R: 79\%) and temporal (L: $12 \%$ and R: 14\%) lobes. The third state (BNS3) was identified over the time period $150-190 \mathrm{~ms}$. It was found to be very similar to the previous network in term of spatial location of nodes. However, a main difference relates to appearance, in BNS3, of nodes located in the right frontal lobe (L: $0 \%$ and $\mathrm{R}: 5 \%$ ). A dominant network (BNS4) was then later identified over the period 190-320 ms. This network is characterized by long-range connections between the occipital (L: $44 \%$ and R: $61 \%$ ) and the frontal lobe (L: 28\% and R: 22\%). This network was then followed by BNS5 over the time period 320-480 ms in which connections with the pre-central region appear (L: $18 \%$ and R: 13\%). Finally, the last network BNS6 was obtained over the period 481-535 ms. This functional network mainly involved the temporal lobe (L: 39\% and R: 31\%) and central region in each hemisphere (L: 26\% and R: 36\%). In addition, our results showed also strong inter-hemisphere connections mainly between the occipital lobes at BNS1, BNS2, BNS3 and BNS4 and between frontal lobes at BNS4 and BNS5. (See figure S2 for multiple view -left, right, top, front and back- of the obtained networks).

The identified BNSs were compared with clusters obtained from null model (surrogate data). Typical examples of the results corresponding to the BNS2 and BNS3 are shown in figure 3. To evaluate the temporal profiles of the identified BNSs, we compared the duration of both networks with those obtained by the 100 surrogates. Figure 3 shows significant differences 
between the duration of clusters identified from surrogates data and the original ones $(p<0.01)$. To evaluate the spatial distributions of the BNSs, we chose to compare the number of nodes identified in the occipital regions (involved mainly in the visual information at this period) for the original and surrogates data. Results also show significant differences $(p<0.01)$ for both BNS2 and BNS3. The same results were obtained for all the other BNSs.

EEG source localization vs. functional connectivity. We compared the above-described source connectivity results with those obtained from the more classical source localization analysis, as reported in Figure 4. The distributions of sources were obtained from dense-EEG recordings using the weighted Minimum Norm Estimate (wMNE) algorithm. They were averaged over subjects and over time, for each of the 6 periods identified with the segmentation algorithm. Results show that both approaches lead to dramatically different results regarding involved brain regions. The main differences are described hereafter. During time periods $\mathrm{t} 1, \mathrm{t} 2$ and $\mathrm{t} 3$, the source localization method disclosed a pronounced bilateral activation of the dorsolateral frontal cortex. Interestingly, this region was not present in the networks associated with the BNSs identified over the same three periods by the source connectivity method. To some extent, the same result holds for $\mathrm{t} 4$, for the right hemisphere. Another discrepancy was observed for the inferior occipital and the occipito-temporal regions that were not disclosed by the wMNE algorithm over period t5 while the same regions were not part of the network associated with BNS5 revealed by the source connectivity method. Finally, over t6, differences between both methods relate to the central and insula regions, as they did not appear in localized sources but were present in the network associated with BNS6.

Spatiotemporal dynamics of brain networks and associated neocortical areas. In order to further precise characterization of the spatiotemporal dynamics of identified networks and in 
order to accurately identify the neocortical areas involved in these networks, we combined a quantitative network measure with the high-resolution anatomical parcellation of the brain surface ( 1000 ROIs). First, the "strength" was used as network measure (high "strength" means "strong" connections at a given node). Second, the parcellation/labelling step allowed us to establish a direct link between spatially distributed nodes showing significantly high strength values $(>$ mean $+1 \mathrm{SD})$, on the one hand, and the corresponding cortical areas (labelled ROIs) on the other hand. Results are presented in figure 5A for the 6 periods identified during the cognitive process. First, it was found that 3 (minimum) to 5 (maximum) brain areas are involved in a given BNS. Second, the highest strength values were observed for t2 (L: $0.85 \pm 0.1$ and $\mathrm{R}: 0.71 \pm 0.1)$ and $\mathrm{t} 3(\mathrm{~L}: 0.9 \pm 0.13$ and $\mathrm{R}: 0.91 \pm 0.12$ ) as compared with t1 (L: $0.3 \pm 0.08$ and R: $0.32 \pm 0.11$ ), t4 (L: $0.42 \pm 0.04$ and R: $0.4 \pm 0.4$ ), t5 (L: $0.21 \pm 0.03$ and R: $0.17 \pm 0.04)$ and t6 (L: $0.39 \pm 0.08$ and R: $0.41 \pm 0.071)$. Third, and very interestingly, the same cortical areas (denoted by the same colors) were found to be present in the networks associated with 2 consecutive BNSs. This is typically the case for the left occipital pole (red color) and the left/right inferior occipital gyrus and sulcus (blue) that were identified at BNS1, BNS2, BNS3 and BNS4. Similarly, the left inferior temporal sulcus (cyan) remained present in networks associated with BNS3 and BNS4 while the anterior occipital sulcus (dark blue) was found to be active during BNS2 and BNS3.

These results suggest that the spatiotemporal brain dynamics of picture naming are characterized by transitions between overlapping networks associated with transiently stable states (from $30 \mathrm{~ms}$ to $160 \mathrm{~ms}$ ), as summarized in Fig. 3B. Indeed, as depicted, four transitions (T1-T4) are characterized by a "continuous" flow of information processing as some network nodes common to consecutive BNSs were revealed by the proposed source connectivity approach. In addition, as detailed in the discussion, we could relate i) the timing of the 6 
periods and ii) the identified networks associated with BNSs to well-established neuropsychological phases of the picture recognition/naming task (visual recognition, memory access and categorization, semantic processing, phonological encoding and articulation).

\section{Discussion}

Appropriate dense-EEG processing reveals the spatiotemporal dynamics of functional brain networks.

To our knowledge, this study is one of the very few attempts to characterize the spatiotemporal dynamics of brain networks over a short duration cognitive task $(<1$ second $)$ from scalp EEG data. Results show that appropriate processing of dense-EEG recordings allows for identification of networks that are in agreement with brain regions involved in the same cognitive task and identified from other modalities (mainly fMRI and PET, (Price, 2012), see below for details). However, these results go beyond those obtained with classical neuroimaging techniques, as the proposed method offers the unique advantage to track the network dynamics with high temporal (in the order of ms) and spatial ( 1000 ROIs) resolution.

The good performance of this processing can be explained by three key steps to obtaining relevant networks, in terms of time and space features. The first step is the reconstruction of cortical sources distributed over a high-resolution mesh by solving the EEG inverse problem. We previously realized a multifactor analysis to analyze the effect of the different factors that intervene in the EEG source connectivity analysis. This methodological study showed that the wMNE algorithm combined with dense electrode array (180 scalp electrodes) leads to optimal results (Hassan et al., 2014). The second step is the estimation of the functional connectivity 
using phase synchronization among gamma oscillations present in the time-course of reconstructed sources. Our results demonstrate that this step is crucial for identifying networks with high specificity in regard to the performed task. The third step is the segmentation, in time, of the cognitive process into brain network states (BNSs). Based on the k-means clustering of brain networks, we have developed an algorithm, originally at scalp EEG level at ms scale (Mheich et al., 2015). This algorithm was used here for the first time on cortical level networks. This segmentation procedure automatically leads to a timing that strongly matches the successive steps previously reported of brain processing from image perception to naming. This 'switching behavior' of the function connectivity networks has been very recently reported for resting states data using modeling approach (Hansen et al., 2014). However, this time varying characteristics of the functional brain networks should be taken with caution as similar behaviors could be observed in random data (noise) as reported in Hlinka (Hlinka \& Hadrava, 2015), hence the importance of testing the results against null models which was performed in our paper.

\section{Source functional connectivity vs. source localization}

A major question that is addressed in this paper relates to the difference between the proposed network-based approach and the previously-used approach aimed at localizing the sources of activated regions during cognitive tasks (Miozzo, Pulvermüller, \& Hauk, 2014; Salmelin, Hari, Lounasmaa, \& Sams, 1994). Although both methodologies (source-based and networkbased) lead to similar results regarding the involvement of the occipital and frontal lobes during the cognitive task, this study shows that the information extracted from dense-EEG recordings in both cases is dramatically different.

Conceptually, the fundamental difference between both approaches is that the source localization totally ignores all possible interactions between brain regions. When performing 
source localization analysis, the sources with highest amplitude (averaged at given time period or computed at the instant of peak amplitude of the signal) were classically retained (Salmelin et al., 1994). However, to some extent (depending on threshold), this approach neglects the possible contribution of "low energy" sources. As shown in the supplementary figure S3, the threshold process can dramatically modify the results of the localization. For instance, when only the highest 50 or $75 \%$ amplitude sources are kept in the wMNE algorithm, the temporal lobe is not retained as being activated although it has been reported to participate in semantic processing in the exact same task (Patterson, Nestor, \& Rogers, 2007; Price, 2012).

Conversely, the hypothesis behind the network-based approach is that sources can be synchronized regardless their amplitude. Our results show that edges represented in identified networks correspond to connections both among sources with high amplitude (occipital) and sources with low amplitude (temporal). We believe that the network-based approach allows revealing networks that are more specific to the performed task. An illustrative example is the absence of the dorsolateral prefrontal cortex in identified networks during the first $200 \mathrm{~ms}$ while this region is particularly active over the entire task in the source-localization approach, probably due to sustained attentional processes that are not strictly related to picture visualization, decoding and naming.

\section{Brain networks involved in picture naming task}

Regarding the successive steps of the picture naming task, our findings corroborate previous studies based on other modalities, mainly fMRI and PET (see (Price, 2012) for review). For the first BNS (BNS1, 0:119 ms), results showed a network involving the inferior occipital, the lateral occipito-temporal sulcus and occipital pole. This period was shown to be related to the visual feature extraction preceding the object category recognition (Thorpe, Fize, \& Marlot, 
1996; Vanrullen \& Thorpe, 2001). Interestingly, the visual features obtained by Vanrullen et al. revealed response peaking at $120 \mathrm{~ms}$ after the onset which is very comparable to our limit for the first BNS (119 ms). For BNS2 (120:150 ms), the identified functional network mainly comprises the occipital regions (the bilateral inferior occipital, the left occipital pole, right anterior occipital and the left middle occipital and Lunatus). These regions are well known to play a capital role in the processing of visual information and object recognition (Engel, König, Kreiter, \& Singer, 1991; Müller \& Gruber, 2001). Moreover, the gamma activity in this time period was shown to marker of object recognition and binding (Martinovic, Gruber, Ohla, \& Müller, 2009; Müller \& Gruber, 2001).

For BNS3 (151:190 ms), results also indicate a mainly occipital network but with an implication of the bilateral inferior temporal sulcus. This system is known to be related to lexical retrieval, lemma retrieval and lemma selection (Indefrey \& Levelt, 2004). It is also involved in semantic working memory system when someone tries to remind the name of the objects (Martin \& Chao, 2001). In their study, the authors show a discrepancy in the temporal lobe involvement for objects versus animals with more activity in the inferior temporal sulcus for objects and in the superior temporal sulcus for animals. Our picture set comprises 39 animals versus 109 objects or non-animal images. The dominant representation of objects in our experimental set could have shaped this part of the graph favoring the inferior temporal sulcus at the expense of the superior temporal sulcus. During BNS4 (191:320 ms), the network involves the left inferior temporal gyrus in addition to the inferior temporal sulcus. These regions were stated to be in direct relation to semantic processing (Martin \& Chao, 2001). It is also the time window in which the N200 classically appear. The N200 is a marker of semantic processing in go/no-go tasks (Thorpe et al., 1996). Together with the appearance at this stage of frontal nodes, we assume that this large BNS could also integrates the access 
to phonological forms during overt naming which has been shown by Graves et al. (Graves, Grabowski, Mehta, \& Gordon, 2007). In a somewhat interesting way, we found that this BNS ends $10 \mathrm{~ms}$ before the syllabification step in the model of Indefrey and Levelt (Indefrey \& Levelt, 2004).

At BNS5 (321:480 ms), the functional network involves the left superior precentral and the right postcentral sulcus along with the left orbital sulci and the left superior insula. This network, near to the sensory-motor cortex was reported to be engaged into phonetic and articulatory process of speech (Dronkers, 1996; Levelt et al., 1998). Finally, for the last BNS (BNS6, 481:535 ms), the network was found at the left insular gyrus, the left inferior insular circular sulcus and the right orbital sulcus. This network is typically associated with the beginning of the naming process and the speech articulation as well as listening to own speech (external self-monitoring) (Indefrey \& Levelt, 2004; Levelt et al., 1998). At this stage, the variability between subject's response latencies has become incredibly strong and those results should be considered with caution. A backward analysis of the EEG records and the corresponding BNSs (not presented here) can give us more precise results about the last graphs.

The occipital cortex remains active during several BNSs.

Strikingly, our results indicated that the occipital visual areas (especially the inferior occipital area) remain active for $400 \mathrm{~ms}$, over the first four BNSs. This is likely not an artifact due to open eyes for instance because left occipital region was found to be inactive for the last BNS whereas the pictures are still present on the screen. Also, it cannot be due to a variable delay in the behavioral response as the timing of the very early steps of the occipital activity for object identification is only very slightly modified due to attentional effects and the $\mathrm{N} 1$ is not modified at all (Mangun \& Buck, 1998). Those regions are part of what the literature calls the 
lateral occipital complex (LOC) (Grill-Spector, Kourtzi, \& Kanwisher, 2001). They are particularly involved in object representation and for analyzing objects shape independently of other low level characteristics such as color texture. Those characteristics not only serve to address the mental lexicon but also form a part of the large-scale network that builds the semantic representation of objects (Grill-Spector et al., 2001; Huth, Nishimoto, Vu, \& Gallant, 2012). Those representations are held in visual working memory during the complete process (Harrison \& Tong, 2009). It could then take part of a large-scale network characterized by high gamma synchronization contributing to information processing during almost the whole cognitive process of naming.

This persistent occipital cortex activation suggests that the ventral stream does not simply correspond to a sequential Feed-Forward activation (Gilbert \& Li, 2013) of involved brain regions but instead, to a resonance process within a large-scale network. From these results, we hypothesize that, in this network, the image is first processed in the occipital areas of the visual cortex, inducing a sustained gamma activity that lasts for on average $400 \mathrm{~ms}$. This oscillatory activity is then progressively and sequentially compared to oscillations in the other areas. Finally, image categorization, memory access and semantic processing emerge from gamma synchronization among involved brain regions. These results are also in accordance with the resonance properties of specific networks of neurons that are able to selectively respond to inputs at preferred frequencies (Akam \& Kullmann, 2014; Hutcheon \& Yarom, 2000)

\section{Methodological considerations}

In the present study, the whole processing was performed on group-averaged data. The connectivity matrix was computed using the phase locking value (PLV) method at each time instant for each subject and all matrices were averaged over the 21 subjects, yielding a group- 
averaged connectivity matrix at each time sample of the post-stimulus response. The segmentation algorithm was applied to this averaged data. The main advantage of this approach is that it preserves the networks common to all subjects of the group and reduces the inter-individual variability. Consequently, results obtained from this analysis represent the spatiotemporal dynamics of networks that occur most constantly within the group of subjects. It is also noteworthy that we assumed i) that there is an anatomical correspondence among subjects (a template 3D mesh was used as the source model) and ii) that the transitions between the functional connectivity states occur in a repeatable manner over subjects. However, the inter-subject variability was not totally ignored in our analysis. The index "network presence" (see supplementary materials) of each BNS was computed among all the subjects. The results show that the first three BNSs have the highest network presence $(80 \%$, $82 \%$ and $81 \%$ for BNS1, BNS2 and BNS3 respectively). Conversely, the network presence values decreased after BNS3 to reach $64 \%, 62 \%$ and $41 \%$ for BNS4, BNS5 and BNS6 respectively. We are aware that due to strong behavioral reaction time discrepancies, the resonance duration of the nodes within the occipital areas over the BNS4 and BNS5 depends on the variability of the cognitive steps that follows the object identification. Those cognitive steps are importantly modulated by attentional resources and the $400 \mathrm{~ms}$ resonance duration before the BNS6 could be due to a mix of long BNS4 in some individuals or for some specific events with short BNS5 of other faster subjects.

Regarding the functional connectivity measure, we used the PLV method (Lachaux et al., 1999) which provides high performance to detect the inter-trial synchronization at each time instant. However, the PLV method requires a relatively high number of stimuli (148 in our case for each subject) to be correctly estimated. Consequently, this method cannot be easily applied to on-going activity, as for the analysis of resting state networks for instance. 
Although EEG source connectivity reduces the problem of field spread to a certain extent, it does not yet, provide a perfect solution. The field spread effect is an open question and unsolved issue and it is indeed one of the main challenges when performing EEG/MEG inverse solution. In the connectivity context, the main effect of the field spread is referred to 'source leakage', which denotes the possible 'artificial' correlations that can exist between very close sources. In our study, we have considerably limited this problem. We firstly decided to use PLV, which has been shown to be very efficient to reduce field spread (REF). The second step is the 'clustering' or the anatomical parcellation, which consist of reconstructing the sources on a high spatial resolution cortex mesh then averaging the dynamics sources located in the same ROIs. The functional connectivity was then computed between these averaged sources. The averaging over ROIs increases the distance between the positions of the sub-regions, which reduces some artificial correlations between very close sources (at the same ROIs). In addition, our results showed high number of long range connections. Very few approaches have been proposed recently to deal with the source leakage by either normalizing the edges weights by the distance between the nodes or removing the very close edges. However, each of these approaches has its advantage and disadvantages and can remove 'real' connections in most cases (REF Shofflen 2009). Nevertheless, it is important to keep in mind that the source reconstruction algorithms can reduce the volume conduction problem but not address it completely.

\section{Conclusion}

We used dense-EEG recordings during a picture naming task to characterize the spatiotemporal dynamics of functional brain networks. We showed that the picture naming task can be divided into six brain network states (BNSs) characterized by significantly high gamma synchronization. Results revealed that fast transitions occur between these BNSs and 
last from $30 \mathrm{~ms}$ to $160 \mathrm{~ms}$. The spatial location of nodes and edges in the identified networks, as well as the precise timing of transitions, show a precise cognitive function (visual recognition, semantic processing and speech processing) can be associated with each network. In addition, networks associated with BNSs partially overlap. We hypothesized that the persistence of several brain regions in successive BNSs participates to fast and efficient information processing in the brain.

\section{Acknowledgements}

This work has received a French government support granted to the CominLabs excellence laboratory and managed by the National Research Agency in the "Investing for the Future" program under reference ANR-10-LABX-07-01. It was also financed by the Rennes University Hospital (COREC Project named conneXion, 2012-14). This work was also supported by the European Research Council under the European Union's Seventh Framework Programme (FP7/2007-2013) / ERC grant agreement $n^{\circ} 290901$. The authors also want to thanks Deok-Hee Kim-Dufor for her help in analyzing the voice onset time of the recorded oral responses.

\section{References}

Akam, T., \& Kullmann, D. M. (2014). Oscillatory multiplexing of population codes for selective communication in the mammalian brain. Nature Reviews Neuroscience, 15(2), 111-122.

Alario, F. X., \& Ferrand, L. (1999). A set of 400 pictures standardized for French: Norms for name agreement, image agreement, familiarity, visual complexity, image variability, and age of acquisition. Behavior Research Methods, 31(3), 531-552.

Allen, E. A., Damaraju, E., Plis, S. M., Erhardt, E. B., Eichele, T., \& Calhoun, V. D. (2012). Tracking wholebrain connectivity dynamics in the resting state. Cerebral Cortex, bhs352.

Boersma, P. (2002). Praat, a system for doing phonetics by computer. Glot international, 5(9/10), 341-345.

Bressler, S. L., \& Menon, V. (2010). Large-scale brain networks in cognition: emerging methods and principles. Trends in cognitive sciences, 14(6), 277-290. 
Dale, A. M., \& Sereno, M. I. (1993). Improved Localization of Cortical Activity by Combining EEG and MEG with MRI Cortical Surface Reconstruction: A Linear Approach. Journal of Cognitive Neuroscience, 5(2), 162-176. doi: doi:10.1162/jocn.1993.5.2.162

Delorme, A., \& Makeig, S. (2004). EEGLAB: an open source toolbox for analysis of single-trial EEG dynamics including independent component analysis. Journal of neuroscience methods, 134(1), 9-21.

Destrieux, C., Fischl, B., Dale, A., \& Halgren, E. (2010). Automatic parcellation of human cortical gyri and sulci using standard anatomical nomenclature. Neuroimage, 53(1), 1-15.

Doesburg, S. M., Roggeveen, A. B., Kitajo, K., \& Ward, L. M. (2008). Large-scale gamma-band phase synchronization and selective attention. Cerebral Cortex, 18(2), 386-396.

Dronkers, N. F. (1996). A new brain region for coordinating speech articulation. Nature, 384(6605), 159-161.

Engel, A. K., König, P., Kreiter, A. K., \& Singer, W. (1991). Interhemispheric synchronization of oscillatory neuronal responses in cat visual cortex. Science, 252(5010), 1177-1179.

Fell, J., \& Axmacher, N. (2011). The role of phase synchronization in memory processes. Nature Reviews Neuroscience, 12(2), 105-118.

Fischl, B. (2012). FreeSurfer. Neuroimage, 62(2), 774-781.

Gilbert, C. D., \& Li, W. (2013). Top-down influences on visual processing. Nature Reviews Neuroscience, $14(5), 350-363$.

Graves, W. W., Grabowski, T. J., Mehta, S., \& Gordon, J. K. (2007). A neural signature of phonological access: distinguishing the effects of word frequency from familiarity and length in overt picture naming. Journal of Cognitive Neuroscience, 19(4), 617-631.

Grill-Spector, K., Kourtzi, Z., \& Kanwisher, N. (2001). The lateral occipital complex and its role in object recognition. Vision research, 41(10), 1409-1422.

Hansen, E. C., Battaglia, D., Spiegler, A., Deco, G., \& Jirsa, V. K. (2014). Functional connectivity dynamics: Modeling the switching behavior of the resting state. NeuroImage.

Harrison, S. A., \& Tong, F. (2009). Decoding reveals the contents of visual working memory in early visual areas. Nature, 458(7238), 632-635.

Hassan, M., Dufor, O., Merlet, I., Berrou, C., \& Wendling, F. (2014). EEG Source Connectivity Analysis: From Dense Array Recordings to Brain Networks. PloS one, 9(8), e105041.

Hlinka, J., \& Hadrava, M. (2015). On the danger of detecting network states in white noise. Frontiers in Computational Neuroscience, 9, 11.

Hutcheon, B., \& Yarom, Y. (2000). Resonance, oscillation and the intrinsic frequency preferences of neurons. Trends in neurosciences, 23(5), 216-222.

Hutchison, R. M., Womelsdorf, T., Allen, E. A., Bandettini, P. A., Calhoun, V. D., Corbetta, M., . . GonzalezCastillo, J. (2013). Dynamic functional connectivity: promise, issues, and interpretations. Neuroimage, $80,360-378$.

Huth, A. G., Nishimoto, S., Vu, A. T., \& Gallant, J. L. (2012). A continuous semantic space describes the representation of thousands of object and action categories across the human brain. Neuron, 76(6), $1210-1224$.

Indefrey, P., \& Levelt, W. J. (2004). The spatial and temporal signatures of word production components. Cognition, 92(1), 101-144.

Lachaux, J.-P., Rodriguez, E., Martinerie, J., \& Varela, F. J. (1999). Measuring phase synchrony in brain signals. Human brain mapping, 8(4), 194-208.

Levelt, W. J., Praamstra, P., Meyer, A. S., Helenius, P., \& Salmelin, R. (1998). An MEG study of picture naming. Journal of Cognitive Neuroscience, 10(5), 553-567.

Logothetis, N. K., Pauls, J., Augath, M., Trinath, T., \& Oeltermann, A. (2001). Neurophysiological investigation of the basis of the fMRI signal. Nature, 412(6843), 150-157.

Mangun, G. R., \& Buck, L. A. (1998). Sustained visual-spatial attention produces costs and benefits in response time and evoked neural activity. Neuropsychologia, 36(3), 189-200.

Martin, A., \& Chao, L. L. (2001). Semantic memory and the brain: structure and processes. Current opinion in neurobiology, 11(2), 194-201.

Martinovic, J., Gruber, T., Ohla, K., \& Müller, M. M. (2009). Induced gamma-band activity elicited by visual representation of unattended objects. Journal of cognitive neuroscience, 21(1), 42-57.

Mheich, A., Hassan, M., Khalil, M., Berrou, C., \& Wendling, F. (2015). A new algorithm for spatiotemporal analysis of brain functional connectivity. Journal of neuroscience methods, 242, 77-81.

Miozzo, M., Pulvermüller, F., \& Hauk, O. (2014). Early parallel activation of semantics and phonology in picture naming: Evidence from a multiple linear regression MEG study. Cerebral Cortex, bhu137.

Müller, M. M., \& Gruber, T. (2001). Induced gamma-band responses in the human EEG are related to attentional information processing. Visual Cognition, 8(3-5), 579-592.

Nunez, P. L. (2006). Electric fields of the brain: the neurophysics of EEG: Oxford University Press. 
Pascual-Marqui, R. D., Michel, C. M., \& Lehmann, D. (1995). Segmentation of brain electrical activity into microstates: model estimation and validation. Biomedical Engineering, IEEE Transactions on, 42(7), 658-665.

Patterson, K., Nestor, P. J., \& Rogers, T. T. (2007). Where do you know what you know? The representation of semantic knowledge in the human brain. Nature Reviews Neuroscience, 8(12), 976-987.

Price, C. J. (2012). A review and synthesis of the first 20years of PET and fMRI studies of heard speech, spoken language and reading. Neuroimage, 62(2), 816-847.

Rodriguez, E., George, N., Lachaux, J. P., Martinerie, J., Renault, B., \& Varela, F. J. (1999). Perception's shadow: long-distance synchronization of human brain activity. Nature, 397(6718), 430-433.

Rubinov, M., \& Sporns, O. (2010). Complex network measures of brain connectivity: uses and interpretations. Neuroimage, 52(3), 1059-1069.

Salmelin, R., Hari, R., Lounasmaa, O., \& Sams, M. (1994). Dynamics of brain activation during picture naming. Nature, 368(6470), 463-465.

Schneider, W., Eschman, A., \& Zuccolotto, A. (2002). E-Prime: User's guide: Psychology Software Incorporated.

Sporns, O. (2010). Networks of the Brain: MIT Press.

Supp, G. G., Schlögl, A., Trujillo-Barreto, N., Müller, M. M., \& Gruber, T. (2007). Directed cortical information flow during human object recognition: analyzing induced EEG gamma-band responses in brain's source space. PloS one, 2(8), e684.

Tadel, F., Baillet, S., Mosher, J. C., Pantazis, D., \& Leahy, R. M. (2011). Brainstorm: a user-friendly application for MEG/EEG analysis. Computational intelligence and neuroscience, 2011, 8.

Thorpe, S., Fize, D., \& Marlot, C. (1996). Speed of processing in the human visual system. nature, 381(6582), 520-522.

Vanrullen, R., \& Thorpe, S. J. (2001). The time course of visual processing: from early perception to decisionmaking. Journal of cognitive neuroscience, 13(4), 454-461. 

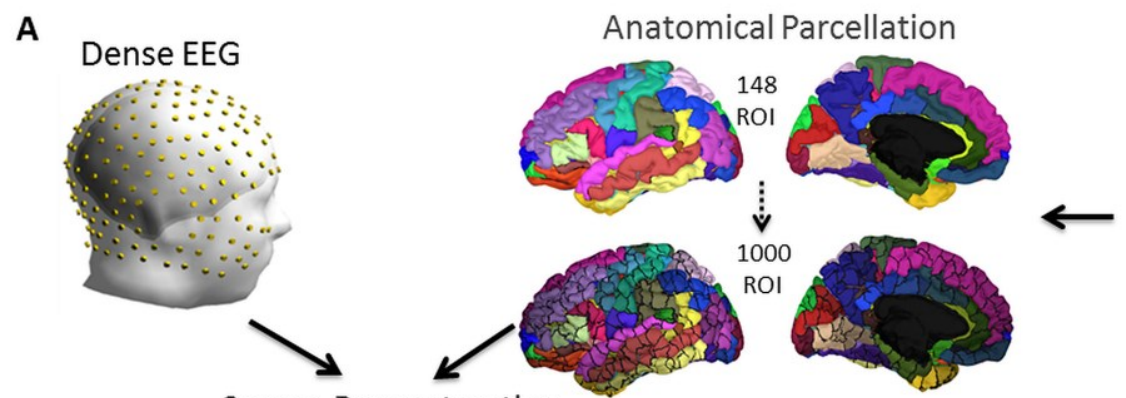

Source Reconstruction

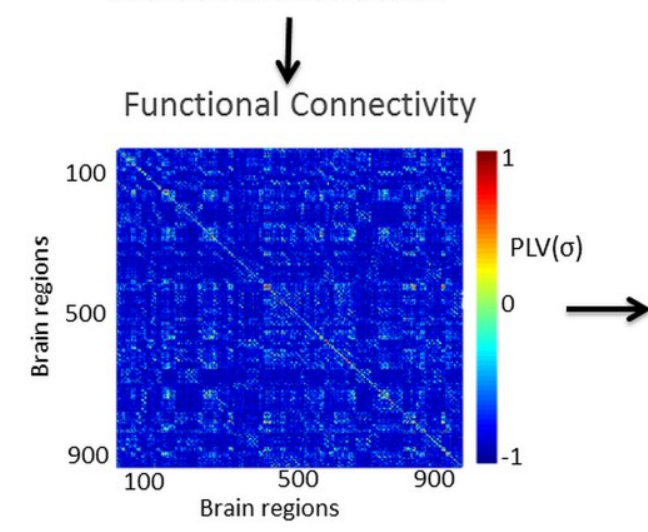

Functional Network

B

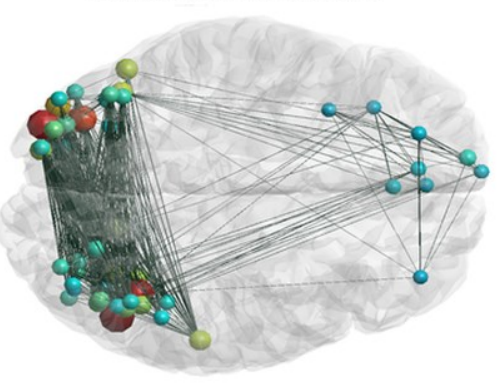

Functional connectivity at millisecond time scale

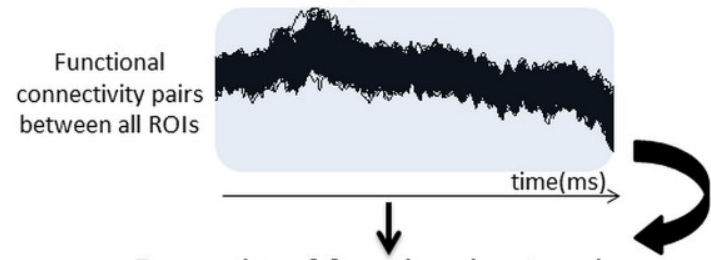

K-means

clustering

Dynamics of functional networks

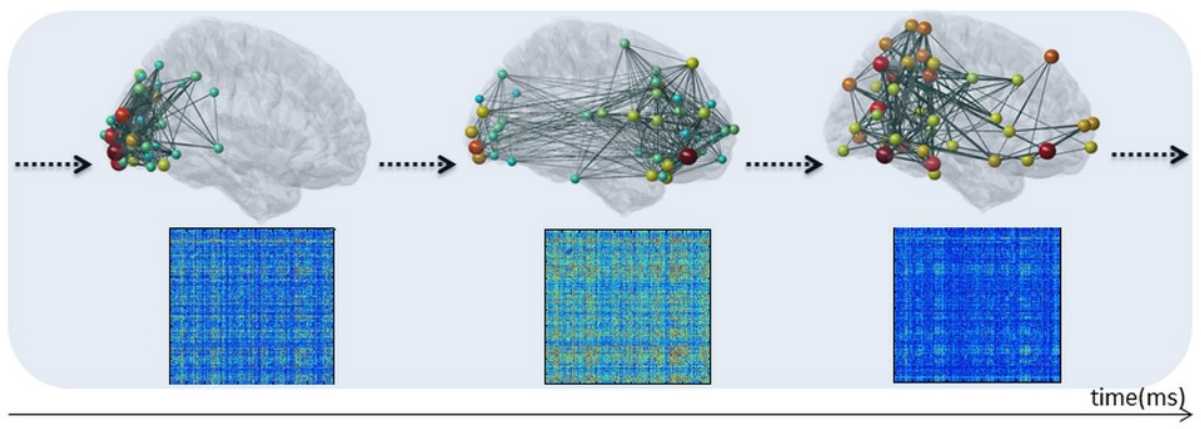


Figure 1:

Structure of the investigation: A) Dense EEG (256 electrodes) were recorded during picture naming task. Structural MRI images were segmented and anatomically parcellated (Destrieux et al., 2010) using Freesurfer (Fischl, 2012) to obtain 148 regions. These 148 regions were subdivided using Brainstorm (Tadel et al., 2011) to obtain higher spatial resolution (about 1000 regions of interests). The inverse problem was then solved using the weighted Minimum Norm Estimate algorithm. The time series of the reconstructed sources were obtained. The functional connectivity between the reconstructed sources was computed using the Phase Locking Value method. A high resolution functional connectivity matrix was obtained and the corresponding functional brain network was visualized. B) This procedure was carried out at millisecond scale and a high number of functional connectivity matrices were obtained. A $K$ means clustering approach was used to obtain the brain network states (BNSs) using a segmentation algorithm (Mheich et al., 2015) summarized in materials and methods section and detailed in the supplementary document. 


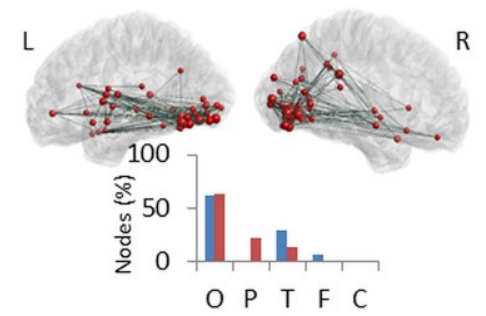

- Left

- Right

t4: 191-320 ms. BNS4
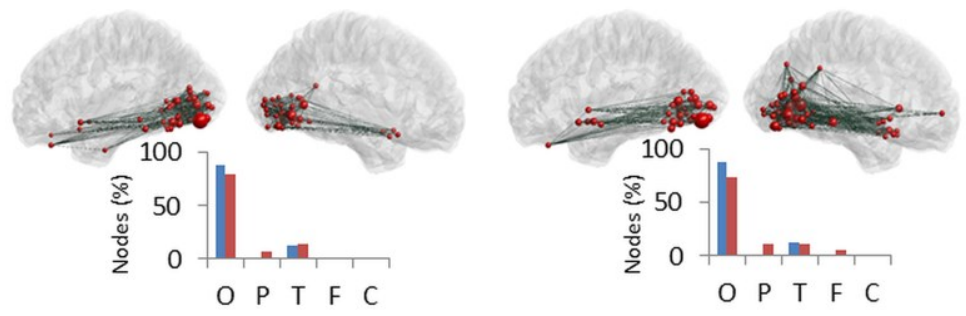

t5: $321-480$ ms. BNS5

t6: 481-535 ms. BNS6
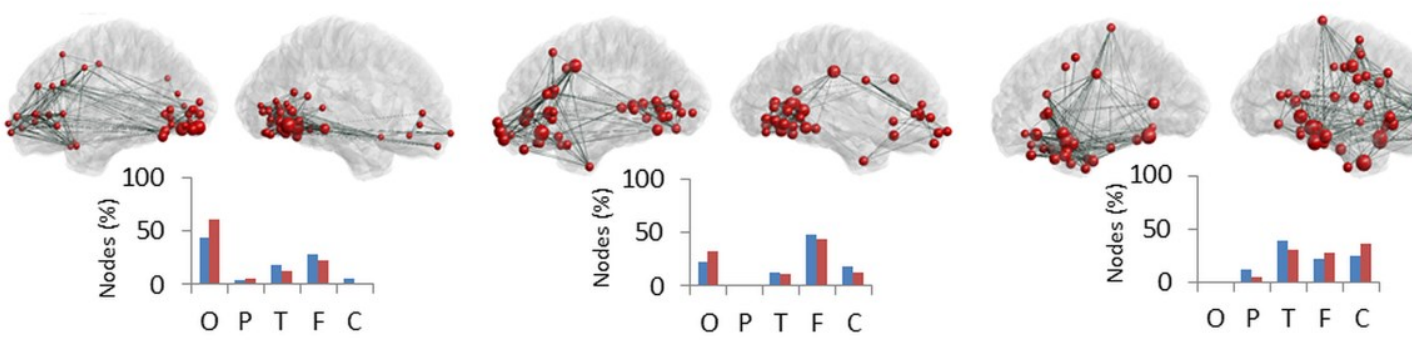

Figure 2:

EEG source connectivity: The exact time periods of the six brain network states identified by the segmentation algorithm are reported (see Materials and Methods for more details about the algorithm). The high resolution networks associated with the BNSs are visualized in left (L) and right (R) view (see figure S2 for other views). Nodes have the same color with different sizes that indicate of the strength value of the node. Edge's thickness represents the connection weight. The networks are 'globally' quantified and the number of nodes in each macroscopic region (O: occipital, $\mathbf{P}$ : parietal, $\mathbf{T}$ : temporal, $\mathbf{F}$ : frontal and $\mathbf{C}$ : central) are presented. 

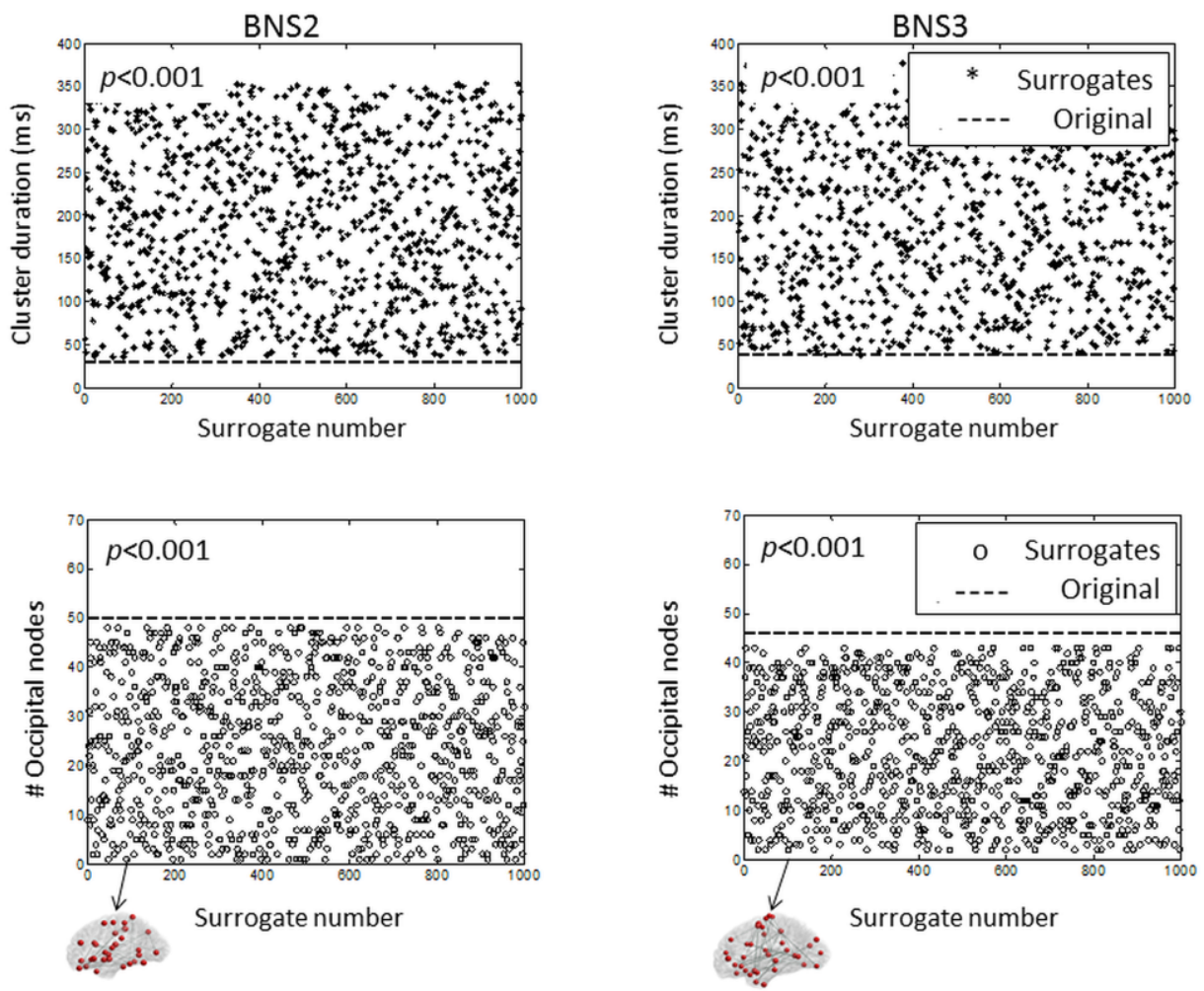

Figure 3:

Statistical analysis: The identified BNSs are compared with null model represented by clusters obtianed from surrogate data. Typical examples of the difference between the temporal profiles (Up) and the spatial distributions (Bottom) of BNS2 (left) and BNS3 (right) and those obtained from surrogates data, are presented. The '*' and 'o' represent the values of the surrogates and the horizontal dashed lines represent the original values. 
C t1: $0-119 \mathrm{~ms}$

$\max$
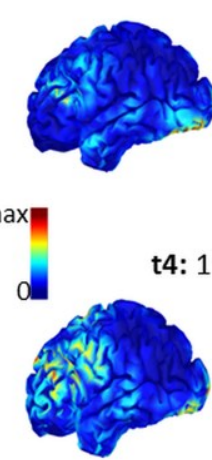

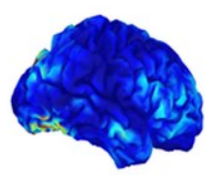

t2: $120-150 \mathrm{~ms}$
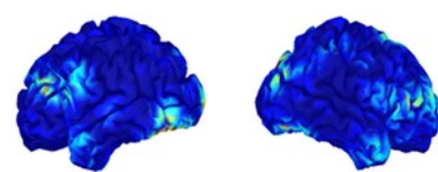

t5: $321-480 \mathrm{~ms}$
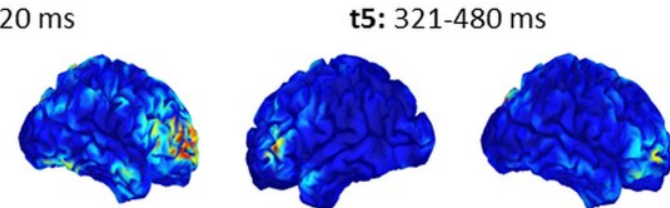

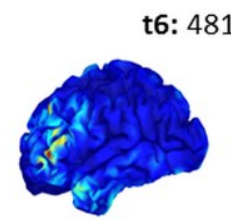

t3: 151-190 ms

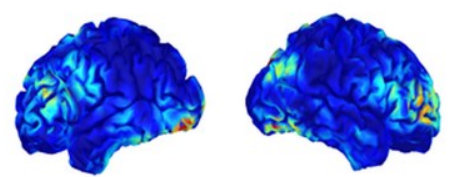



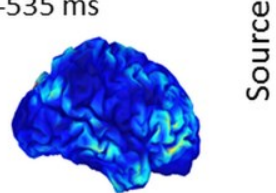

Figure 4:

EEG source localization: For the same time periods identified for the BNSs, the results of the source localization using wMNE are depicted. The sources were averaged at each time period. Red color represents the sources with the highest amplitude. 


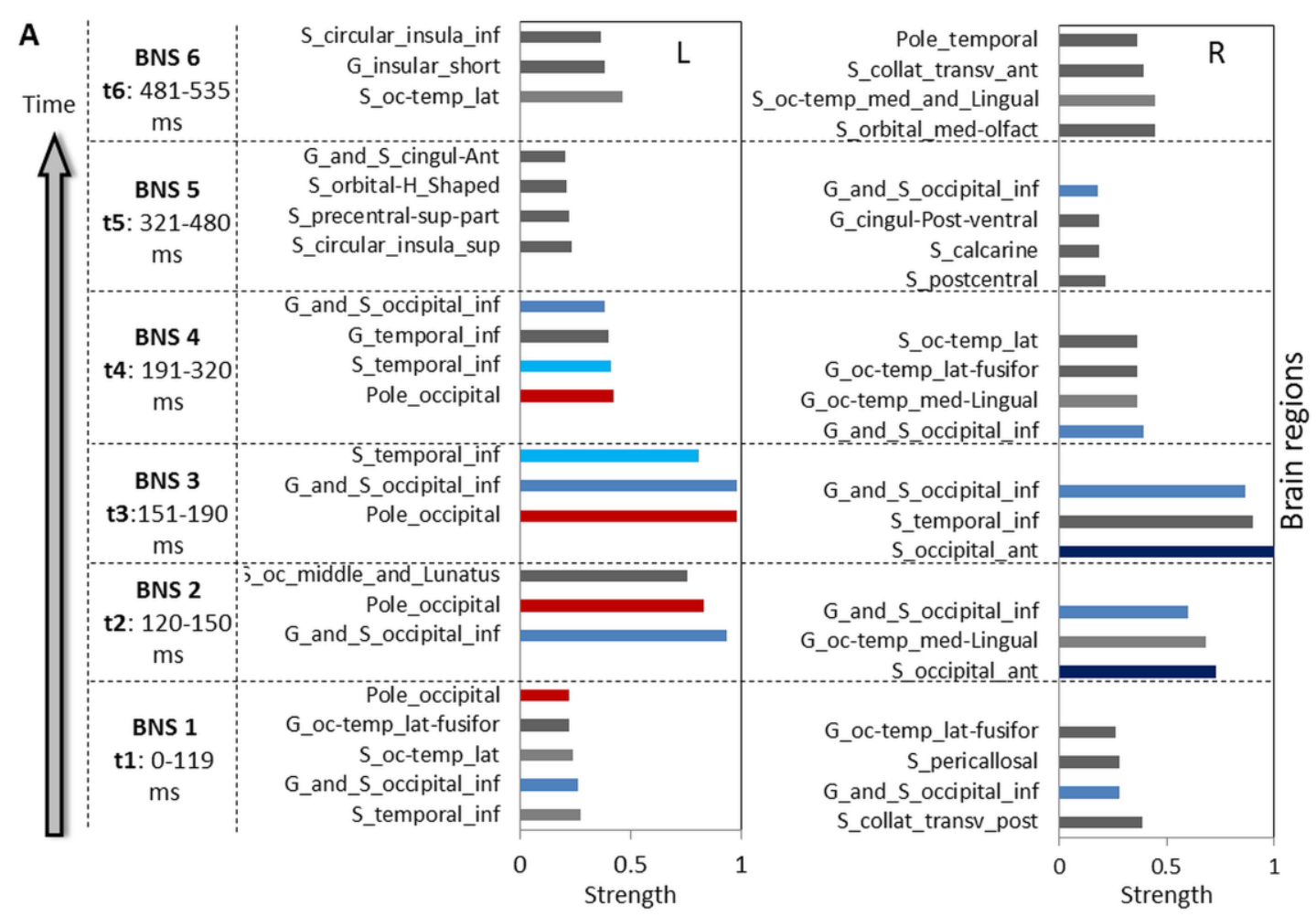

B R S

L G

Anterior occipital Inferior temporal

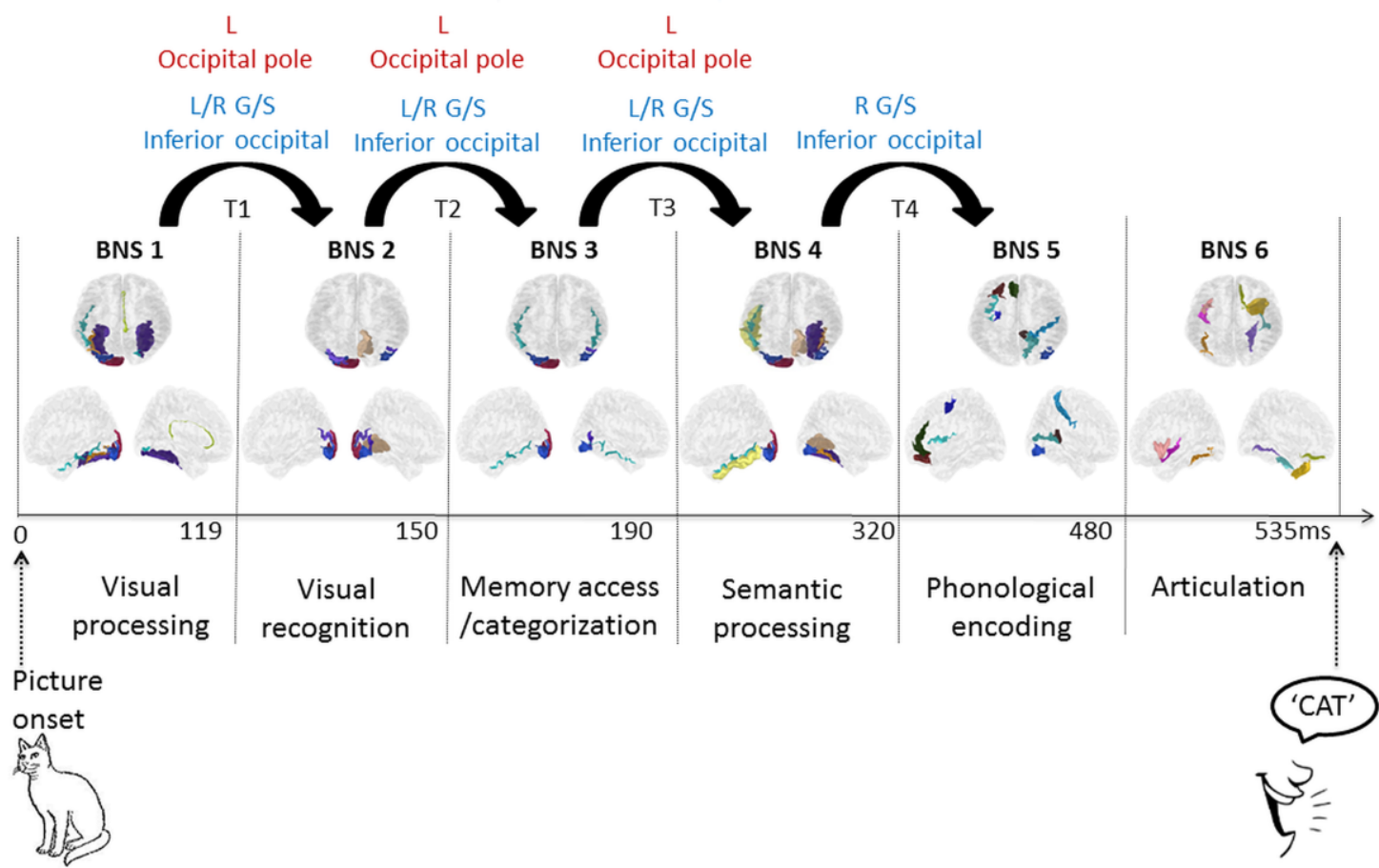


Figure 5:

Spatiotemporal dynamics of brain networks and associated neocortical areas: A) The strength values of each node were calculated for all networks associated with the BNSs. The identified nodes (ROIs) were retained only if they provide significant strength value (> mean+1SD). The common ROIs between two consecutive BNS have the same color. B) The identified regions in part A were color-coded based on the anatomical parcellation of Destrieux Atlas (Destrieux et al., 2010) using Brainstorm Tool (Tadel et al., 2011). The underlying cognitive functions for each BNS are presented. For each transition (T) between two consecutive BNS, the common regions are reported. Abbreviations: L: left, R: Right, G: gyrus, S: Sulcus. 